\title{
Markencontrolling - Sicherstellung der Effektivität und Effizienz der Markenführung
}

1. Markencontrolling als Teilbereich des Marketingcontrollings

1.1 Marketingcontrolling als Sicherstellen der Rationalität einer marktorientierten Unternehmensführung

1.2 Markencontrolling als spezifische Controllingperspektive

2. Messung des Markenwissens

2.1 Quantitative Methoden zur Messung des Markenwissens

2.2 Qualitative Methoden zur Messung des Markenwissens

3. Messung des Markenwertes

3.1 Notwendigkeit und Nutzen der Markenbewertung

3.2 Verfahren zur Messung des Markenwertes

3.3 Grundprobleme der Markenwertmessung

3.4 Integration des Markenwerts in das Markencontrolling

4. Messung der Wirkungen von Markenstärke und -wert

5. Mehrdimensionale Ansätze des Markencontrollings

6. Markenspezifisches Marketingaccounting

7. Fazit

Literaturverzeichnis

Prof. Dr. Torsten Tomczak ist Inhaber des Lehrstuhls für Betriebswirtschaftslehre, insbesondere Marketing sowie Direktor des Instituts für Marketing und Handel (IMH) an der Universität St. Gallen. Dr. Sven Reinecke ist Dozent für Betriebswirtschaftslehre an der Universität St. Gallen und Dr. Philomela Kaetzke ist Wissenschaftliche Assistentin am Institut für Marketing und Handel (IMH) an der Universität St. Gallen. 



\section{Markencontrolling als Teilbereich des Marketingcontrollings}

\subsection{Marketingcontrolling als Sicherstellen der Rationalität einer marktorientierten Unternehmensführung}

Controlling lässt sich nicht mit Rechnungswesen gleichsetzen. Schwerpunktverlagerungen in der Praxis (Horváth 1998, S. 75) zeigen, dass Controller keine (rein) historisch orientierten Buchhalter (Registratoren) mehr sind, sondern der zukunfts- sowie aktionsund somit managementorientierte Teil ihrer Tätigkeit zunimmt.

In neueren Veröffentlichungen legen Weber und Schäffer (1998) die Basis für einen integrierenden Rationalitätssicherungsansatz des Controllings, der sich auf das Marketingcontrolling übertragen lässt. Weber (1999, S. 30ff.) strukturiert den Führungsprozess idealtypisch (vgl. Schaubild 1). Ausgangspunkt ist die Willensbildung, die reflexiv oder intuitiv ablaufen kann. Erfolgt sie reflexiv, so muss dazu ausreichendes, einer analytischen Betrachtung zugängliches Wissen verfügbar sein. Bei einer intuitiven Willensbildung ist der Prozess weniger transparent und noch wenig erforscht, häufig aber dennoch sehr effizient.

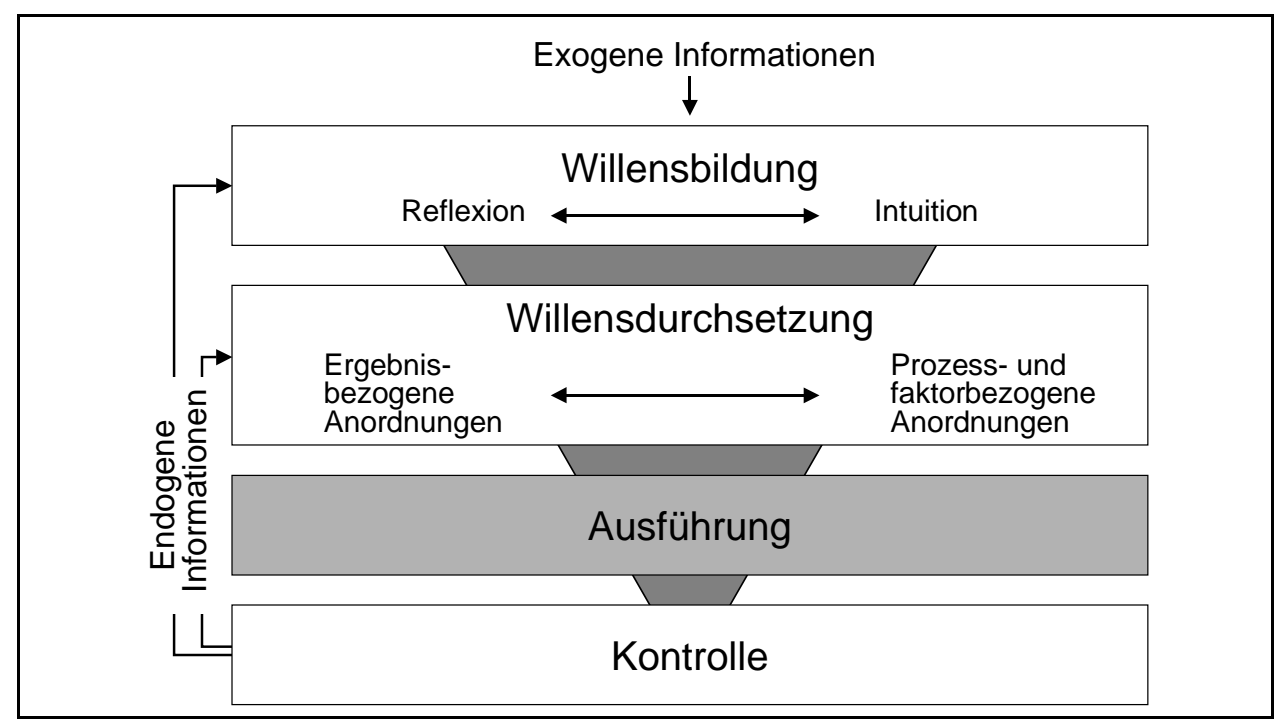

Schaubild 1: Idealtypischer Führungsprozess

(Quelle: Weber 1999, S. 33) 
Um den Willen durchzusetzen, muss dieser den ausführenden Stellen übermittelt werden. Dies kann durch ergebnis-, prozess- oder faktorbezogene Anordnungen erfolgen. Idealtypischerweise wird der kommunizierte Wille vom Ausführungssystem umgesetzt. Diese Phase ist allerdings nicht Teil des Führungssystems, wohl aber die Kontrolle der Übereinstimmung zwischen Gewolltem und Erreichtem. Das Ergebnis dieses SollIst-Vergleichs führt entweder zu einer erneuten Willensbildung (z.B. einer Planrevision) oder fließt erneut in die Willensdurchsetzung ein (beispielsweise Anordnung konkreter Tätigkeiten, um eine zukünftige Übereinstimmung von Soll und Ist zu erreichen). Willensbildung, -durchsetzung und Kontrolle sind somit eng miteinander vernetzt.

Nach Weber ist es die Aufgabe des Controllings, die Rationalität und somit die Effektivität und Effizienz der Führung sicherzustellen. Controlling lässt sich somit auch als eine spezifische Form der Qualitätssicherung der Führung bzw. des geschilderten Führungsprozesses interpretieren (Weber 1999, S. 38). Schaubild 2 reflektiert marketingbezogen die vier zentralen „Rationalitätsengpässe“ (Weber/Schäffer 1998, S. 22, 2001):

1. Marketingcontrolling muss gewährleisten, dass das Management mit den erforderlichen Informationen aus dem eigenen Unternehmen und bezüglich Kunden, Konkurrenz und Marktpartnern versorgt wird. Im Informationszeitalter steht dabei das rechtzeitige Erkennen von Technologie- und Marktentwicklungen im Mittelpunkt.

2. Marketingcontrolling muss sicherstellen, dass in der Phase der Willensbildung Reflexion (Business-Pläne, Investitionsrechnungen usw.) und Intuition (beispielsweise das Gespür für den Markt) in einem ausgewogenen Verhältnis stehen. Zwischen Planung und Kreativität besteht ein fruchtbares Spannungsfeld.

3. Neben der Koordination der Instrumentevielfalt muss das Marketingcontrolling insbesondere ein durchgängiges Marketingführungssystem und somit eine reibungslose Marketingimplementierung gewährleisten. Das Überwachen des Marketing im Rahmen von Kontrollen ist ein unverzichtbarer, wenn auch bisher vernachlässigter Bereich des Marketingcontrolling.

4. Marketingcontrolling berührt maßgeblich Aspekte der Personalführung (Anreizgestaltung, Mitarbeiterselektion) und der Organisation (Kompetenzverteilung); daher kann es nicht - wie teilweise in der Literatur üblich - allein auf das Planungs-, Informations- und Kontrollsystem beschränkt werden.

Marketingcontrolling umfasst somit die Sicherstellung von Effektivität und Effizienz aller Marketingmaßnahmen. Es darf sich nicht darauf beschränken, lediglich Konzepte des Controllings unreflektiert auf das Marketing zu übertragen; vielmehr muss es für das Marketing ein weitgehend eigenständiges, problembezogenes Instrumentarium entwickeln, das insbesondere die Kunden- und Konkurrenzorientierung, die Sachziel- und Potenzialorientierung als auch die Durchgängigkeit eines marktorientierten Führungssystems berücksichtigen muss (Meffert 1982, S. 100ff.; Reinecke 2003). 


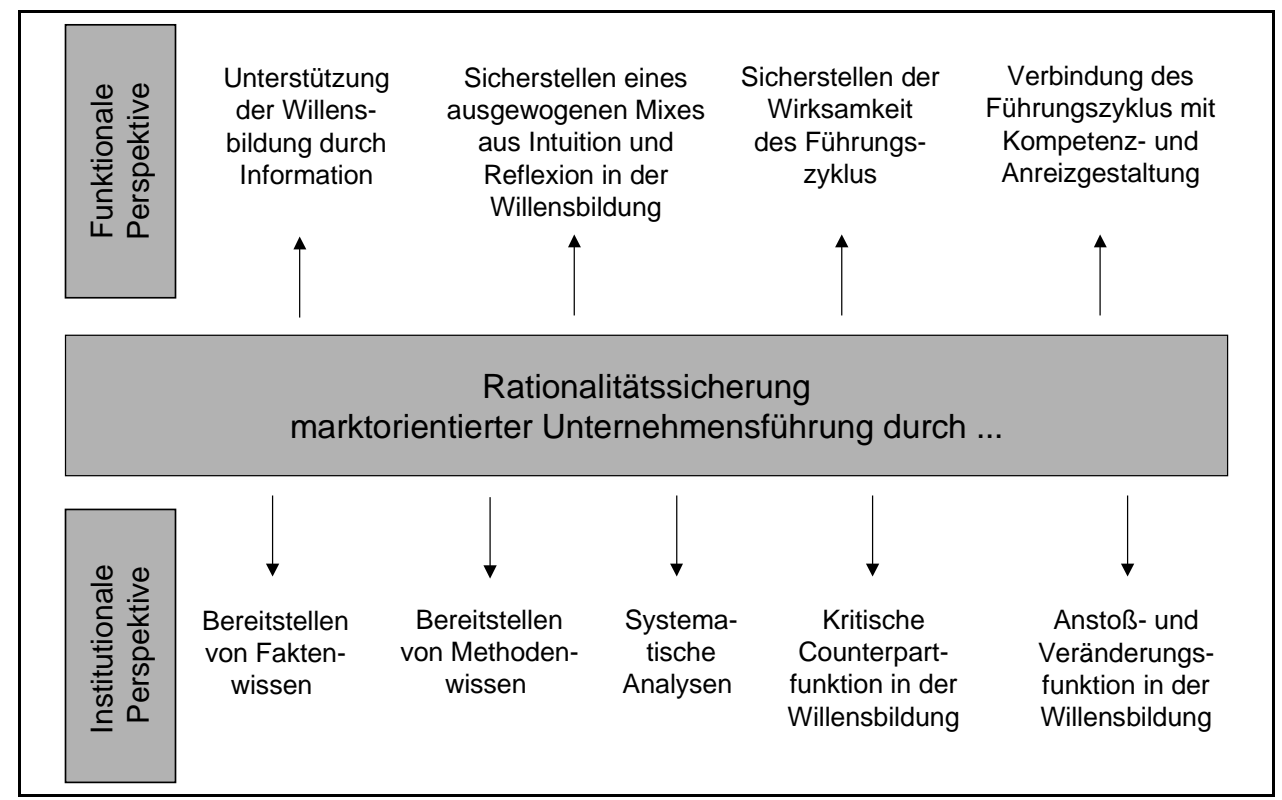

Schaubild 2: Sicherung der Rationalität marktorientierter Unternehmensführung (Quelle: In Anlehnung an Weber 1999, S. 40)

\subsection{Markencontrolling als spezifische Controllingperspektive}

Markencontrolling kann als eine (Teil-)Perspektive auf das Marketingcontrolling interpretiert werden. Alle Aspekte des Marketingcontrollings, die markenrelevant sind, sind somit auch Teil des Markencontrollings. Nachfolgend wird eine „Marke“ als Gesamtwahrnehmung einer markierten Angebotsleistung durch den Kunden verstanden (Schiele 1997, S. 9f.; ähnlich Meffert 2000, S. 847).

Die Bedeutung der Marke zeigt sich insbesondere, wenn man sie als Ressource auffasst, um nachhaltige Wettbewerbsvorteile zu erzielen. Eine Marke erzeugt unternehmerischen Mehrwert, indem sie Risiken reduziert (beispielsweise durch eine geringere Empfänglichkeit von Kunden gegenüber Konkurrenzmaßnahmen), Cash-Flows beschleunigt (z.B. durch eine verbesserte Handelsunterstützung bei Markenausdehnungen sowie eine schnellere Innovationsdiffusion), Cash-flows durch Mehreinnahmen erhöht (u.a. aufgrund einer unelastischeren Preiselastizität), Cash-Flows durch Ausgabeneinsparungen erhöht (zum Beispiel aufgrund einer höheren Effektivität und Effizienz der Marketingkommunikation) oder den Restwert der Markeninvestitionen am Ende des Betrachtungszeitraums erhöht (Srivastava et al. 1998). 
Im Folgenden steht das funktionale Markencontrolling im Vordergrund - unabhängig davon, wer diese Aufgaben durchführt. Je nach Unternehmensgröße wird ein Großteil dieser Markencontrollingaufgaben häufig nicht von spezialisierten Controlling- oder Marktforschungsabteilungen, sondern vielmehr vom Markenmanagement selbst und somit von Linienverantwortlichen erledigt. In Analogie zu den dargelegten „Rationalitätsengpässen" muss sich das Markencontrolling insbesondere folgenden Aspekten widmen:

Informationsversorgung: Neben der Bereitstellung und Aufbereitung klassischer Marktforschungsinformationen (beispielsweise aus Haushalts- und Handelspanels) sowie markenbezogener Umsatz- und Ergebnisgrößen stehen hier zum einen die Frage nach der Stellung der Marken bei Kunden (Markenbekanntheit und -image) sowie zum anderen konkurrenzbezogene Vergleiche im Mittelpunkt.

- Sicherstellen der Ausgewogenheit zwischen Reflexion und Intuition: Das Markencontrolling muss seine Aufgabe als „kritischer Counterpart“ wahrnehmen, um beispielsweise sicherzustellen, dass Entscheidungen bezüglich Markenerweiterungen, -transfers, -lizensierungen, -restrukturierungen, -verkäufen oder -fusionen nicht ausschließlich intuitiv erfolgen, sondern vielmehr analytisch fundiert werden.

- Sicherstellen eines durchgängigen Markenführungssystems: Das Markencontrolling hat sicherzustellen, dass die strategische Markenplanung mit der operativen Umsetzung und somit auch der Budgetierung koordiniert wird. So ist zu gewährleisten, dass die Aufteilung der Budgets auf Push- und Pull-Maßnahmen nicht nur die kurzfristigen Absatz-, sondern auch die langfristigen Markenziele berücksichtigt. Insbesondere sind für Markenplanung und -führung ausgewogene monetäre und nicht-monetäre Kenngrößen zu bestimmen (Stichwort: Marken-Balanced-Scorecard, vgl. Meffert/ Koers 2001; Brand Score Card, vgl. hierzu auch den Beitrag von Linxweiler/Brucker in Teil 7 dieses Handbuches). Ferner muss das Markenmanagement mit den anderen Bereichen abgestimmt werden; so sind beispielsweise Kunden- und Markendeckungsbeitragsziele zu koordinieren. Weiterhin sind Kontrollen unverzichtbar: Ziel ist es, die Ergebnisse der Markenführungsstrategien und -maßnahmen hinsichtlich Effektivität (Wirksamkeit) und Effizienz (Wirtschaftlichkeit) zu überprüfen.

- Verbindung mit der Kompetenz- und Anreizgestaltung: Eine wichtige Fragestellung des Markencontrollings ist beispielsweise, welche Kompetenzen ein nationaler Marken- bzw. Product Manager hat. Werden ihm lediglich finanzielle Ergebnisziele vorgegeben (Umsätze, Deckungsbeiträge, Marktanteile) oder auch differenzierte Markengrößen (Bekanntheitsgrad im Zielsegment, Markenimage, Kundenloyalität)? Oder werden die Markenmanager sogar input- bzw. prozessorientiert gesteuert (beispielsweise durch eine zentrale Vorgabe von Werbemitteln und -plänen sowie standardisierte, internationale Marktforschungsinstrumente)? 


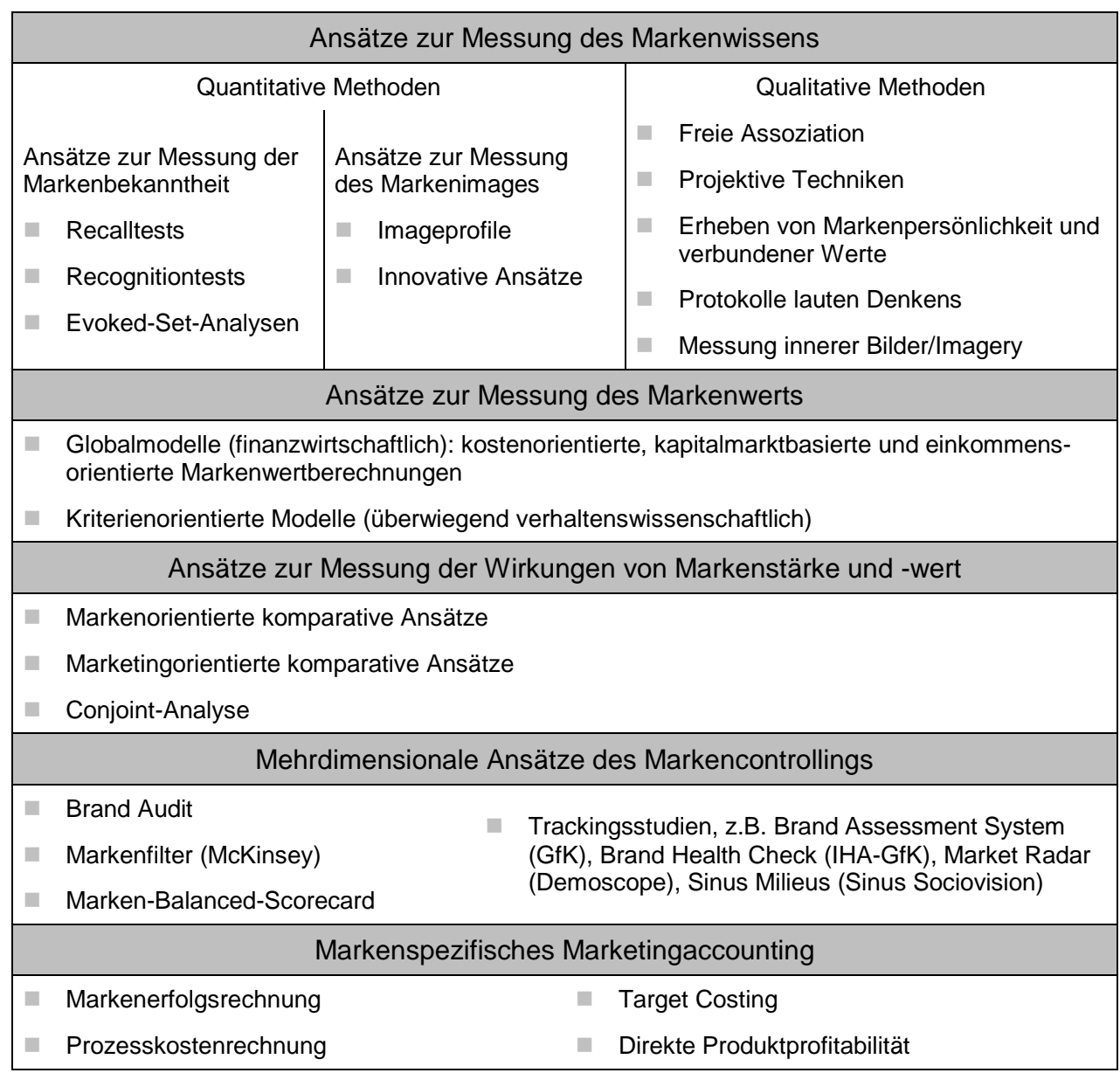

Schaubild 3: Ausgewählte Ansätze des Markencontrollings im Überblick (Quelle: In Anlehnung an Keller 1998, 2001)

Schaubild 3 bietet einen Überblick über die nachfolgend erläuterten Ansätze des Markencontrollings (Keller 1998, 2001), die dabei helfen, die definierten Rationalitätsengpässe zu überwinden. 


\section{Messung des Markenwissens}

Verhaltenswissenschaftliche Ansätze (Aaker 1991; Keller 1993, 2001; Esch/Geus 2001, S. 1031ff.) gehen davon aus, dass eine Marke am Markt erfolgreich ist, wenn Konsumenten sich von ihr ein bestimmtes, mit positiven Assoziationen verbundenes Bild machen. Im Folgenden sollen das Markenwissen der Konsumenten, die einzelnen Dimensionen dieses Wissens und Methoden zu dessen Messung behandelt werden.

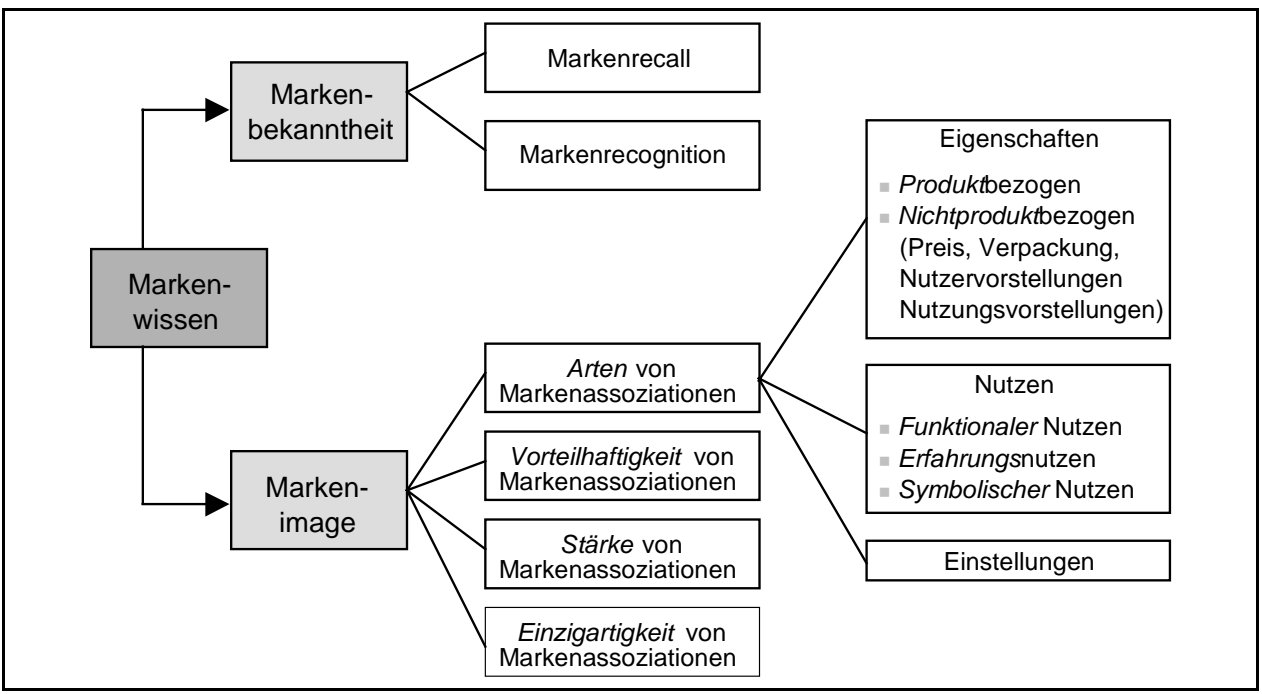

Schaubild 4: Operationalisierung des Markenwissens

(Quelle: In Anlehnung an Keller 1993, S. 7)

Das Markenwissen der Konsumenten (vgl. Schaubild 4) lässt sich anhand zweier Dimensionen - Markenbekanntheit und Markenimage - beschreiben bzw. operationalisieren (Esch/Geus 2001, S. 1033ff.; Keller 2001, S. 1061ff.). Die Markenbekanntheit wird definiert als die „Fähigkeit des Verbrauchers, die Marke unter verschiedenen Bedingungen zu erinnern oder wiederzuerkennen" (Keller 2001, S. 1061). Man unterscheidet hierbei zwischen dem Markenrecall (der aktiven Markenbekanntheit) und der Markenrecognition (der passiven Markenbekanntheit). In der Regel bezeichnet man die Markenbekanntheit als notwendige Bedingung für die Bildung eines spezifischen Images der Marke in den Köpfen der Konsumenten. Bei geringem Involvement und ähnlichen Images der verschiedenen Marken kann Markenbekanntkeit unter Umständen sogar eine positive Kaufentscheidung bewirken (Esch/Geus 2001, S. 1032; Keller 2001, S. 1061). In vielen Fällen ist die Markenbekanntheit jedoch keine hinreichende, sondern lediglich 
eine notwendige Bedingung dafür, dass eine Marke im Kaufentscheidungsprozess bevorzugt berücksichtigt wird. Daneben spielt das Markenimage eine entscheidende Rolle.

Das Markenimage bezeichnet die „Wahrnehmung und Bevorzugung einer Marke auf der Basis verschiedener gespeicherter Markenassoziationen“ (Keller 2001, S. 1061). Bei der Bildung von Markenvorstellungen spielen bildliche Informationen häufig eine wichtigere Rolle als verbale Informationen, weil das Gedächtnis für verbale Informationen als leistungsfähiger gilt (Paivio 1971, S. 381f.; Kroeber-Riel/Weinberg 1999, S. 345ff.). Insgesamt lässt sich das Markenimage anhand mehrerer Dimensionen charakterisieren (Esch/Geus 2001, S. 1033f.; Keller 2001, S. 1062f.):

- Arten der Markenassoziationen: Markenassoziationen können auf konkreten produktbezogenen oder nicht produktbezogenen Eigenschaften, aber auch auf dem Nutzen der Marke oder bestimmten Einstellungen basieren. Beispielsweise beruht das Image der Marke Milka auf bestimmten produktbezogenen Eigenschaften wie etwa dem zarten Schmelz der Schokolade. Darüber hinaus sind auch nicht produktbezogene Eigenschaften wie die lilafarbene Verpackung und die Vorstellung einer intakten Alpenlandschaft bedeutsam für das Image der Marke Milka.

- Vorteilhaftigkeit der Markenassoziationen: Assoziationen, die die Marke gegenüber anderen Marken positiv abheben, können zu einem prägnanten Markenimage beitragen. Zu beachten ist, welche Assoziationen bzw. deren Ausprägungen aus Sicht der Konsumenten als relevant und positiv eingeschätzt werden.

- Stärke der Markenassoziationen: Markenmanager können die Assoziationen und damit auch das Markenimage stärken, indem sie darauf achten, den Konsumenten ein im Zeitablauf möglichst konsistentes Bild der Marke zu liefern. Darüber hinaus kann sich auch ein hohes Involvement der Konsumenten positiv auf die Stärke der Markenassoziationen auswirken.

- Einzigartigkeit von Markenassoziationen: Wenn sich eine Marke durch eine herausragende Eigenschaft abhebt, trägt dies dazu bei, dass die Marke im Kaufentscheidungsprozess der Konsumenten weniger austauschbar erscheint.

\subsection{Quantitative Methoden zur Messung des Markenwissens}

Nachfolgend werden zunächst ausgewählte quantitative Methoden zur Messung des Markenwissens (Markenbekanntheit und -image) behandelt (Esch/Geus 2001, S. 1035ff.; Keller 2001, S. 1063), die im Rahmen des Markencontrolling zum Einsatz kommen.

Zur Messung der Markenbekanntheit eignen sich Recall- und Recognitiontests. Ziel von Recalltests ist es, die aktive Markenbekanntheit zu bestimmen (Kroeber-Riel/Weinberg 1999, S. 354; Keller 2001, S. 1069f.). Entsprechend werden ungestützte Erinnerungs- 
messungen eingesetzt: Konsumenten werden aufgefordert, aus dem Gedächtnis die ihnen bekannten Marken einer bestimmten Kategorie anzugeben. Recognitiontests dienen dazu, die passive Markenbekanntheit zu ermitteln (Kroeber-Riel/Weinberg 1999, S. 354; Keller 2001, S. 1069f.). Bei derartigen Tests wird den Konsumenten in der Regel eine Liste mit Marken(-namen) einer bestimmten Kategorie vorgelegt und sie werden gebeten, die ihnen bekannten Marken zu markieren. Der Einsatz von Recognitiontests empfiehlt sich insbesondere (aber nicht ausschließlich), wenn ein relativ geringes Involvement vorliegt und daher die Entscheidung für eine bestimmte Marke in der Regel erst unmittelbar am Point of Sale (PoS) getroffen wird. Wenn es sich jedoch um Kaufentscheidungen mit relativ hohem Involvement handelt, beziehen die Konsumenten bereits verschiedene Marken in ihren Entscheidungsprozess ein, bevor sie eine Einkaufsstätte aufsuchen. Unter dieser Ausgangsbedingung eignen sich eher Recalltests, da hierbei überprüft wird, an welche Marken sich die Konsumenten ohne Vorgabe von Markennamen oder -logos aus dem Gedächtnis erinnern (Kroeber-Riel/Weinberg 1999, S. 383; Keller 2001, S. 1068f.).

Eine weitere Möglichkeit ist die Durchführung so genannter Evoked-Set-Analysen, die Bekanntheits- und Imagemessungen mittels Befragung und Beobachtung kombinieren (Spiggle/Sewall 1987, S. 100); sie folgen einem klassischen „Hürdenprozess”: Von allen verfügbaren Angeboten (Total Set) sind für den Kunden nur jene Angebote bzw. Eigenschaften relevant, die er auch wahrnimmt; er kann sie nur wahrnehmen, wenn er davon Kenntnis hat (Awareness Set). Der Kunde entwickelt zu diesen Angeboten gewisse Einstellungen aufgrund seiner kaufentscheidungsrelevanten Bedürfnisse (Evoked Set). Von den Angeboten werden wiederum nur einige ausgewählt (Action Set).

Die Erhebung von Imageprofilen stellt die bekannteste Methode zur Messung des Markenimages dar. Konsumenten werden gebeten, Marken einer bestimmten Kategorie anhand einer vorgegebenen Liste von Assoziationen zu beurteilen. Solche Imageprofile werden häufig zum Controlling der Markenpositionierung eingesetzt. Bevor derartige Untersuchungen durchgeführt werden können, ist es erforderlich, die relevanten Assoziationen zusammenzustellen, die Zielgruppe zu bestimmen (um geeignete Kunden für die Befragung auswählen zu können) und zu ermitteln, welche Marken in der betrachteten Kategorie in Konkurrenz zueinander stehen (Aaker 1991, S. 137ff.). Auf Basis von Kunden- und Konkurrenzbetrachtung ergibt sich eine längere Liste von Attributen; dabei sollten nach Trommsdorff und Paulssen (2001, S. 1144) bei der Erhebung von Imageprofilen nur solche Markenassoziationen herangezogen werden, die

- für die Bildung von Präferenzen für bestimmte Marken und für den Kaufentscheidungsprozess relevant sind,

durch den Einsatz der Marketinginstrumente beeinflussbar sind sowie

- tatsächlich differenzieren, d.h., die Marke von der Konkurrenz abheben.

Imageanalysen nach dem dargestellten Verfahren weisen im Vergleich zu qualitativen Verfahren den Vorteil auf, dass sie sich auf die zentralen Assoziationen konzentrieren 
und repräsentativ erhoben werden können. Zudem lassen sich die Ergebnisse anschaulich grafisch darstellen. Die Defizite entsprechen teilweise denen des „klassischen“ Positionierungsmodells (Trommsdorff/Paulssen 2000, S. 1056). So sind die herangezogenen Assoziationen für die verschiedenen Marken häufig nicht von gleich hoher Relevanz. Zudem werden Wechselwirkungen zwischen konkurrierenden Marken kaum erfasst: Ist eine Marke beispielsweise hinsichtlich einer relevanten Assoziation aus Konsumentensicht besonders stark, so sind Konkurrenzmarken häufig gezwungen, auf andere Assoziationen auszuweichen. Des Weiteren lassen sich keine Rückschlüsse ziehen, wie sich das Imageprofil einer Marke auf die Veränderung von Markenpräferenzen und auf die tatsächlichen Kaufentscheidungen der Konsumenten auswirkt.

Innovative quantitative Ansätze versuchen, die Defizite klassischer Imagemessungen zumindest teilweise auszugleichen (Esch 2001; Trommsdorff/Paulssen 2001, S. 1149ff.). Beispielsweise ermöglicht der so genannte LOCATOR-Ansatz (Biel 2001, S. 82ff.), Prognosen darüber zu treffen, wie die Markenpräferenzen - und damit letztlich das Kaufverhalten - der Konsumenten durch bestimmte Imageprofile bzw. Änderungen der Imageprofile beeinflusst werden. Basis des Ansatzes ist ein so genanntes Mikromodell. Dabei geht die Untersuchung von einer individuellen Betrachtung des Markenimages aus. Zunächst wird ermittelt, welche Assoziationen in der betrachteten Kategorie relevant sind; basierend auf den Ergebnissen der Befragung einer Gruppe von Konsumenten wird eine Grafik zu diesen Assoziationen entwickelt, die darstellt, wie die in der Kategorie konkurrierenden Marken wahrgenommen werden. Dieselbe Gruppe wird in einer zweiten Befragung zu ihren Präferenzen befragt. Die Konsumenten werden gebeten, eine bestimmte Anzahl von Punkten ihren Präferenzen entsprechend auf die Marken einer Kategorie zu verteilen. Mittels Simulationsrechnungen lassen sich UrsacheWirkungs-Zusammenhänge zwischen Markenimages und -präferenzen ableiten. Der LOCATOR-Ansatz liefert somit beispielsweise Empfehlungen, welche Assoziationen Markenmanager stärken sollten, um die Präferenz für ihre Marke zu erhöhen.

\subsection{Qualitative Methoden zur Messung des Markenwissens}

Neben den quantitativen Ansätzen existieren verschiedene qualitative Methoden zur Messung des Markenwissens. Gemeinsam ist diesen Verfahren, dass sie besonders detaillierte Erkenntnisse über die emotionalen und kognitiven Prozesse liefern, die bei den Konsumenten bei der Bildung und Veränderung von Markenassoziationen ablaufen. In der Regel werden keine repräsentativen Ergebnisse angestrebt. Vielmehr werden qualitative Methoden häufig für Fragestellungen zur Vorbereitung (beispielsweise zur Auswahl von Assoziationen für klassische Imagemessungen; Keller 2001, S. 1064) oder zur Vertiefung der Erkenntnisse quantitativer Untersuchungen eingesetzt.

Bei der Methode der freien Assoziation (Aaker 1991, S. 137f.; Keller 2001, S. 1064f.) sollen Konsumenten ihren Gedanken freien Lauf lassen und spontan angeben, was ihnen 
zu bestimmten Marken einfällt. Ergänzend können vertiefende Fragen gestellt werden. Eine weitere Möglichkeit besteht darin, Konsumenten zu bitten, einen typischen Benutzer der betreffenden Marke zu charakterisieren und zu beschreiben, bei welchen Gelegenheiten er die Marke normalerweise nutzt bzw. konsumiert. Das primäre Ziel der Methode der freien Assoziation ist es, möglichst unverfälschte und detaillierte Aussagen darüber zu erhalten, wie Konsumenten Marken wahrnehmen und welche positiven und negativen Assoziationen sie mit den Marken jeweils verbinden.

Der Einsatz direkter Befragungsmethoden zur Messung des Markenwissen der Konsumenten ist nicht immer sinnvoll. So können oder wollen Konsumenten in einigen Situationen keine Angaben über ihre Einstellungen zu Marken machen; beispielsweise, weil sie ihnen nicht bewusst sind oder weil sie der sozialen Erwünschtheit widersprechen. Zur Überwindung dieser Barrieren eignen sich indirekte, projektive Techniken (Aaker 1991, S. 137ff.; Keller 2001, S. 1065ff.).

Wertvolle Aussagen über die Markenwahrnehmung und das Markenwissen der Konsumenten liefern Untersuchungen zur Markenpersönlichkeit und den entsprechenden Werten (Aaker 1991, S. 137ff.; Keller 2001, S. 1067). Bei dieser Methode erhalten Konsumenten die Aufgabe, sich vorzustellen, dass die Marke eine Person sei. Sie werden dazu aufgefordert, diese Persönlichkeit möglichst ausführlich zu charakterisieren (Aussehen, Kleidung, persönliche Werte, Beruf, soziales Verhalten usw.). Die Ergebnisse derartiger Befragungen liefern häufig Rückschlüsse darauf, wie Konsumenten die Marke erleben und wie sie ihre Beziehung zur Marke sehen.

Detaillierte Informationen über die Markenwahrnehmung der Konsumenten lassen sich durch Protokolle lauten Denkens gewinnen (Grunert 1990; Esch/Geus 2001, S. 1037f.). Beispielsweise werden Konsumenten gebeten, neben sprachlichen auch bildliche Assoziationen und emotionale Eindrücke (beispielsweise Genuss, Lebensfreude, Sicherheit) zur betreffenden Marke zu beschreiben. Dadurch sollen möglichst viele Informationen über die unterschiedlichen Dimensionen des Markenwissens (sprachlich, bildlich, emotional, Markenvergleiche usw.) gewonnen werden. Die Ergebnisse lassen sich dazu nutzen, die Positionierung der Marke zu überprüfen sowie Vergleiche zwischen dem Wissen der Konsumenten über die eigene Marke und über konkurrierende Marken bzw. über die Kategorie allgemein anzustellen.

Der Ansatz zur Messung innerer Bilder (Imagery) konzentriert sich auf die bildlichen Gedächtnisstrukturen der Konsumenten über Marken (Ruge 1988; Kroeber-Riel/ Weinberg 1999, S. 344ff.; Bekmeier-Feuerhahn 2000, S. 1023ff.; Esch/Geus 2001, S. 1039ff.). Bei der Messung innerer Bilder wird primär deren Vividness (Lebendigkeit) und somit deren Klarheit, Deutlichkeit (Kroeber-Riel/Weinberg 1999, S. 344) bzw. Zugriffsfähigkeit (Ruge 1988, S. 100; Bekmeier-Feuerhahn 2001, S. 1116) erhoben. Daneben eignen sich folgende Dimensionen: Einzigartigkeit, Anziehungskraft, Aktivierungsstärke, psychische Nähe, Intensität, Qualität. Zur Erhebung visueller Markenvorstellungen können sowohl verbale Skalen als auch Bilderskalen eingesetzt werden. 
Zusammenfassend lässt sich feststellen, dass in der Regel eine Kombination quantitativer und qualitativer Methoden zu empfehlen ist, um möglichst umfassende und repräsentative, aber auch profunde und unverfälschte Informationen über das Markenwissen der Konsumenten zu erhalten.

\section{Messung des Markenwerts}

Der Markenwert misst das Erfolgspotenzial einer Marke. Trotz seiner Bedeutung wird dieser Begriff jedoch sehr unterschiedlich verwendet. Die verschiedenen Definitionen von Markenwert sind insbesondere auf die unterschiedlichen Ziele der Bewertung von Marken zurückzuführen. Während einige Autoren eine monetäre Bewertung im Sinne eines Bilanzwertes anstreben, legen andere Wissenschaftler den Fokus eher auf die Markensteuerung und versuchen daher, Ursache-Wirkungs-Zusammenhänge durch umfangreiche Kriterienlisten abzubilden.

Das Marketing Science Institute hat versucht, eine integrierende, tragfähige Definition zu entwickeln: „Brand equity can be defined as the set of associations and behavior on the part of a brand's customers, channel members, and parent corporation that permits the brand to earn greater volume or greater margins than it could without the brand name; brand equity gives the brand a strong, sustainable, and differentiated advantage over competitors. This definition implies that brand equity involves a number of dimensions including perceptual or affective measures (for example, impressions of differentiation, image, strength of attachment), behavioral measures (willingness to pay more for the brand, unwillingness to switch, brand share), and financial measures (stability of income stream, above-average price margins, minimal drops in sales after advertising or sales promotion reductions)" (zitiert nach Leuthesser 1988, S. 31).

Diese Definition unterstreicht, dass der Markenwert letztlich auf einer einstellungs- und verhaltensorientierten Kundensicht beruht. Andererseits impliziert der Ausdruck „Wert“, dass diese Potenzialgröße in geeigneter Form finanzwirtschaftlich operationalisiert und möglichst dynamisch quantifiziert wird. Ein Modell von Srivastava und Shocker (1991) verbindet die verhaltens- und finanzwirtschaftliche Sicht. Die Autoren entwickelten die Definition des Marketing Science Institute weiter und unterscheiden im Rahmen eines übergeordneten Brand-Equity-Konzepts die beiden Begriffe „Markenstärke“ (Brand Strength) und „finanzieller Markenwert“" (Brand Value):

„Brand strength may be defined as the set of associations and behaviors on the part of a brand's customers, channel members, and parent corporations that permits the brand to enjoy sustainable and differentiated competitive advantages (i.e. brand strength results in barriers to competition and, therefore, some degree of monopolistic power to the firm controlling the brand)" (Srivastava/Shocker 1991, S. 9). Die 
Markenstärke hängt ab von der derzeitigen Performance und Profitabilität einer Marke, der Verlässlichkeit der Gewinnerwartungen und den Ausbau- und Wachstumspotenzialen. Diese drei Treiber werden wiederum insbesondere durch verhaltenswissenschaftliche Größen (z.B. Bekanntheit, Wertschätzung, Loyalität und Assoziationen) bestimmt. Ferner wirken spezifische Branchenfaktoren - wie z.B. die Konkurrenzintensität - auf die Markenstärke.

„Brand value is the financial outcome of management's ability to leverage brand strength via tactical and strategic actions in providing superior current and future profits and lowered risks. As such, it depends on the ,fit ' of the brand with the firm's objectives, resources (including synergy with other products), and competitive market conditions" (Srivastava/Shocker 1991, S. 9). Der finanzielle Markenwert hängt somit nicht nur von der Markenstärke, sondern insbesondere von der Fähigkeit des Managements ab, mit Hilfe geeigneter Maßnahmen die Markenstärke auch in einen höheren Unternehmenswert (durch höhere und sicherere Geldflüsse) umzusetzen. Daher muss eine finanzielle Messung unternehmensspezifisch aus der Sicht des Eigentümers oder eines potenziellen Käufers erfolgen (Bekmeier-Feuerhahn 1998). So kann eine bestimmte Marke für ein anderes Unternehmen einen höheren finanziellen Wert haben, weil es über andere Ressourcen (z.B. eigene Distributionskanäle), Kompetenzen oder eine breitere Kundenbasis verfügt.

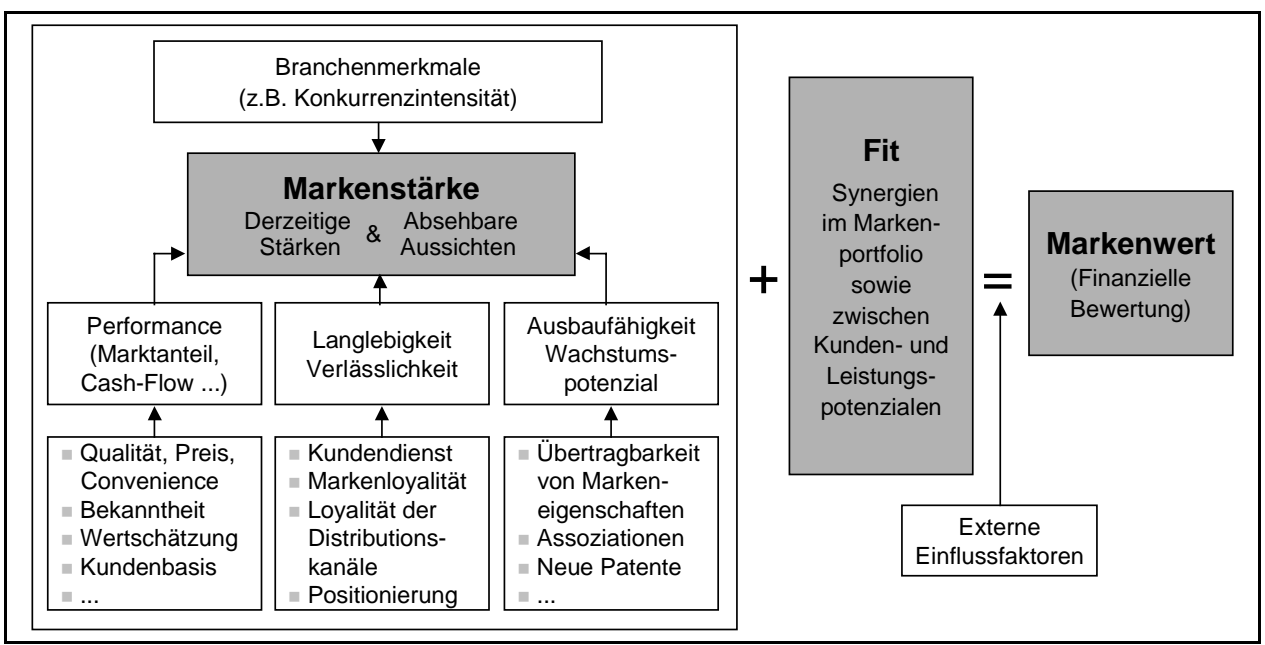

Schaubild 5: Markenwert und Markenstärke (Quelle: In Anlehnung an Srivastava/Shocker 1991, S. 7)

Das Modell deckt sich mit Forschungen im deutschsprachigen Raum, beispielsweise mit Ergebnissen von Bekmeier-Feuerhahn (1998). Auch Meffert und Koers (2001, S. 299f.) 
bauen auf dieser Terminologie auf. Eine besondere Stärke des Konzepts ist die Brückenbildung zwischen dem verhaltens- und wettbewerbsorientierten Marketing sowie dem am Shareholder Value orientierten Finanzwert (vgl. Schaubild 5).

Der Markenwert (Brand Equity) wird nachfolgend als finanzwirtschaftlich bewertetes Ergebnis einer vom Management aktiv gestalteten Markenstärke verstanden.

\subsection{Notwendigkeit und Nutzen der Markenbewertung}

Für Markenbewertungen existiert eine Vielzahl von Anlässen, beispielsweise der Kauf eines Unternehmens, die Bestimmung der Höhe von Lizenzgebühren, die Aktivierung von Marken in Bilanzen sowie die Planung, Steuerung und Kontrolle von Marken (Kriegbaum 2001, S. 77ff.). Nachfolgend steht der Controllinganlass im Mittelpunkt. Der Nutzen von Markenbewertungen für Marketingplanung und -controlling ist in der Marketingwissenschaft unumstritten (Aaker 1996, S. 314ff.):

- Markenstärke als Tracking-Größe ergänzt quantitative Marktgrößen wie Umsatz, Absatz sowie Marktanteil ideal (Franzen 1995, S. 563) und drückt u.a. Veränderungen bezüglich Markenloyalität und Marktpositionierung aus (Cravens/Guilding 1999, S. 56). Erfolgt die Bewertung finanziell, so ist eine Verbindung zum Konzept des Unternehmenswertes möglich.

- Markenbewertungen helfen bei Budgetallokationen, sind eine geeignete Grundlage für die Steuerung eines Markenportfolios (Meffert 1999) bzw. für die Optimierung von Handelssortimenten (Franzen 1995, S. 563) und ermöglichen ein Benchmarking mit der Konkurrenz.

Letztlich ist insbesondere der Prozess der Markenbewertung an sich wertvoll. Daraus ergibt sich ein Erkenntnisgewinn bezüglich des Markenbildungsprozesses. Ferner können die Größen im Rahmen von Investor-Relations-Maßnahmen genutzt werden.

Guilding und Pike (1994, S. 243ff.) untersuchen die verhaltenswissenschaftlichen und organisatorischen Implikationen der Messung des Markenwertes: Aus welchen Gründen sollten Führungskräfte danach streben, den Wert von Marken zu analysieren? Die empirische Überprüfung zahlreicher Hypothesen auf der Basis einer Stichprobe von 140 Führungskräften ergibt, dass der Nutzen der Markenbewertung eindeutig auf langfristig-strategischer, weniger jedoch auf operativer Ebene gesehen wird. Dieses Ergebnis wird ebenfalls von Kriegbaum (2001, S. 160) bestätigt. Ferner zeigt sie, dass Marketingführungskräfte deutlich mehr Nutzen in einer Markenbewertung sehen als Führungskräfte aus den Bereichen Finanzen/Controlling. Dies belegt die internen politischen Ziele einer Markenbewertung, d.h., Marketingführungskräfte hoffen, dadurch ihre Interessen besser vertreten zu können (Guilding/Pike 1994, S. 248ff.). 


\subsection{Verfahren zur Messung des Markenwertes}

Es existiert kein allgemeines Markenbewertungsverfahren, sondern eine Vielzahl unterschiedlicher Methoden. Grundsätzlich lassen sich zwei Grundformen unterscheiden: Globalmodelle und kriterienorientierte Modelle (Güldenberg/Franzen 1992, S. 38).

Globalmodelle versuchen, den (finanziellen) Wert einer Marke als Ganzes zu quantifizieren (vgl. Schaubild 6):

- Kostenorientierte Verfahren sind rückwärtsgerichtet und können eine zukunftsgerichtete Markenbewertung kaum gewährleisten.

- Kapitalmarktbasierte Verfahren sind zwar zukunftsorientiert, allerdings fragwürdig, solange Märkte für Marken nicht effizient sind. Ineffizienzen führen zu enormen und sachlich nicht zu rechtfertigenden Schwankungen.

- Einkommensorientierte Markenwertberechnungen schätzen den finanziellen Wert einer Marke. Untersuchungen in Großbritannien ergaben beispielsweise, dass der durchschnittliche „Mehrwert“ der jeweils führenden Konsumgütermarke gegenüber einer Handelsmarke 40 Prozent und gegenüber der zweitstärksten Marke 10 Prozent beträgt (Crimmins 1992, S. 17). Die Verfahren geben allerdings allein kaum Gestaltungshinweise im Sinne einer Ursache-Wirkungs-Analyse. Außerdem weisen sie das Problem der Reziprozität auf: Die Einschätzung zukünftiger Marketingmaßnahmen wirkt sich auf den heutigen Markenwert aus (Agarwal/Rao 1996, S. 237ff.). Ferner treten Probleme bei der Prognose des Zahlungsstroms, bei der Definition des betrachteten Zeithorizonts und bei der Bestimmung des Abzinsungsfaktors auf (Herrmann 1998, S. 493; Sattler 2000, S. 225ff.).

Kriterienorientierte Modelle versuchen, die Markenstärke zu bewerten (vgl. Schaubild 7), wobei nur wenige Ansätze die Markenstärke auch finanziell quantifizierten. Bei diesen Verfahren stellt sich die Frage, ob die in den einzelnen Markenwertmodellen verwendeten Indikatoren sowie deren Gewichtung prinzipiell tragfähig und insbesondere auf die unternehmensspezifische Situation anwendbar sind (Esch/Andresen 1994, S. 217; Wiedmann 1994, S. 1320). Auch die Anzahl der eingesetzten Kriterien variiert stark. Agarwal und Rao (1996, S. 238ff.) konnten zwar zeigen, dass die zehn wichtigsten Indikatoren für den Markenwert konvergieren, dass aber dennoch alle nötig seien, um Kaufentscheidungen möglichst vollständig zu erklären. Je stärker die Aggregation, desto stärker sind daher die Zweifel, ob ein zusammenfassender Markenwert(-index) bzw. Aussagen über dessen Entwicklung ausreichen, um konkrete Handlungsempfehlungen abzuleiten (Wiedmann 1994, S. 1320; Keller 1998, S. 365). 


\section{Kostenorientierte Verfahren: Grundidee und Bewertung}

Bewertung einer Marke auf Basis einer Kalkulation der mit dieser Marke verbundenen Kosten:

Bewertung der Aufbaukosten (Kosten, die mit dem Aufbau einer Marke verbunden gewesen sind).

Schätzung der Ersatzkosten (Kosten, die damit verbunden wären, einen vergleichbaren Namen und ein vergleichbares Geschäft aufzubauen).

\section{Kapitalmarktbasierte Verfahren: Grundidee und Bewertung}

Diese Verfahren versuchen zu evaluieren, zu welchem Preis eine Marke verkauft werden könnte.
Rückwärtsgerichtet; ungenügende valide Informationen über historische Kosten.

Kostenzurechnung schwierig; Problem der Trennung der Marketingkosten von anderen Kosten.

- Replikation einer Marke häufig aufgrund der Marktkapazität nicht möglich, ohne den Wert bestehender Marken zu beeinflussen.

Einkommensbasierte Verfahren: Grundidee und Bewertung

Diese Verfahren versuchen, den Markenwert aufgrund von Präferenzen zu berechnen, die nicht auf Produktunterschiede zurückzuführen sind:

- Ermittlung diskontierter Cash-FlowUnterschiede zwischen Marken- und Nichtmarkenprodukten durch Kalkulation von Absatzmengenunterschieden und des Preispremiums.

- Schätzung von Gewinnverlusten aufgrund von Präferenzdifferenzen, die entstehen, wenn Markennamen weggelassen würden.

- Bewertung diskontierter (potenzieller) Lizenzeinnahmen.

Schaubild 6: Globalmodelle zur Markenwertmessung

(Quellen: Aaker 1991; Srivastava/Shocker 1991; Simons/ Sullivan 1993; Keller 1998, S. 349ff.; Sattler 1998; Cravens/Guilding 1999, S. 58f.; Baumgarth 2001, S. 236)
- Preispremium häufig praktikable, verständliche und daher akzeptierte Lösung.

Differenzschätzung in Märkten ohne Nichtmarkenprodukte kaum möglich.

- Preispremiummodell führt in Märkten mit ähnlichen Preisen zur systematischen Überbewertung kleiner Präferenzmarken und Unterbewertung von Preis-Mengen-Marken; Marktabgrenzungsproblem.

- Trotz Diskontierung statische Modelle, die weder Markensteuerung noch mögliche Markenerweiterungen berücksichtigen.

Bestimmung von Zeithorizont, Zinssatz und Prognose des Zahlungsstroms bei CashFlow-Berechnungen problematisch. 
Kriterienorientierte Modelle: Ausgewählte Verfahren und Bewertung

- Interbrand-Modell (Ward 1989; Aaker 1991, S. 29; Keller 1998, S. 363; Cravens/ Guildung 1999, S. 59f.): Multiplikatormodell, bei dem der operative Gewinn mit einem branchenspezifischen Multiplikator gewichtet wird. Die Multiplikatorhöhe ergibt sich aus einer Bewertung der Dimensionen der Markenstärke im Vergleich zur Konkurrenz: Marktführerschaft (Leadership), Stabilität, Markt, Internationalität, Trend, kontinuierliche Marketingunterstützung, juristischer Markenschutz. Nichtmarken-Einflussfaktoren wie z.B. das Distributionssystem werden herausgerechnet.

Nielsen-Brand-Monitor (Franzen 1995, S. 564): Berücksichtigung kundenorientierter Größen: Marktattraktivität (Marktvolumen, -wachstum), Durchsetzungsstärke der Marke im Markt (Entwicklung des Marktanteils), Handelsakzeptanz (numerische und gewichtete Distribution), Konsumentenakzeptanz (Bekanntheit, Relevant Set).

icon Brand Trek-Modell (Esch/Andresen 1994, S. 217ff.): Verhaltenswissenschaftliche Messung des Markenwerts im Gedächtnis der Konsumenten mittels Markenbild (Marken-Awareness, Klarheit, Attraktivität des inneren Markenbildes, Eigenständigkeit, Einprägsamkeit, wahrgenommener Werbedruck) und Markenguthaben (Markensympathie, Markenvertrauen).

"Markenwissen“ nach Keller (1993, S. 7): Markenkenntnis (Wiedererkennung, Erinnerung) und -image (Arten, Vorteilhaftigkeit, Stärke, Einzigartigkeit der Assoziationen).

Brand Asset Valuator von Young \& Rubicam (2000): Hierarchisches Vier-Kriterien-Modell: Differenzierung (Basis), Relevanz (Markenbedeutung), Wertschätzung (Qualität, Popularität), Wissen (Verständnis der Markenpersönlichkeit).

- Brand Equity Ten nach Aaker (1996, S. 318ff.): Preispremium, Zufriedenheit/Loyalität, wahrgenommene Qualität, Führerschaft/Popularität, wahrgenommener Wert, Unternehmensassoziationen, Markenpersönlichkeit, -bekanntheit (Wiedererkennung, Erinnerung, Top of Mind, Markendominanz, -familiarität, -wissen), Marktanteil, Preis, Distributionsgrad.

\begin{tabular}{|l|}
\hline \multicolumn{1}{|c|}{ Bewertung von Kriterienmodellen } \\
\hline In der Regel hohe Praktikabilität und gute Eignung für Portfoliomodelle. \\
Grundannahmen der Modelle sind häufig stark vereinfachend und selten empirisch \\
gestützt, ungenügende Operationalisierung der Konstrukte. \\
Zum Teil mangelnde Konsistenz, subjektive Kriterienauswahl und -gewichtung. \\
Mit Ausnahme von Interbrand selten finanzwirtschaftliche Quantifizierung.
\end{tabular}

Schaubild 7: Kriterienorientierte Modelle zur Markenwertmessung 


\subsection{Grundprobleme der Markenwertmessung}

Es stellt sich die Frage, warum zahlreiche Unternehmen trotz des geschilderten Nutzens ihre Marken nicht bewerten. Von 79 Unternehmen, die Marken nicht bewerten, gaben in einer Studie von Kriegbaum (2001, S. 162) 36,7 Prozent an, dass keine geeignete Bewertungsmethode vorhanden sei. 31,9 Prozent beurteilten eine Bewertung zwar als sinnvoll, aber als zu kosten- und/oder zeitintensiv, und 19 Prozent sahen keinen sinnvollen Grund für eine Markenbewertung. Im Rahmen einer Analyse der verschiedenen Formen der Markenbewertung ergeben sich folgende vier zentrale Herausforderungen:

1. Isolierbarkeit/Abgrenzbarkeit einzelner Marken: Einige der Bewertungsverfahren setzen voraus, dass einer Marke Kosten und Erträge zugeordnet werden können. Sobald ein Unternehmen allerdings mehr als eine Marke anbietet, ist eine saubere Zurechnung der Gemeinkosten in der Realität kaum möglich (Aaker 1991, S. 20; Srivastava/Shocker 1991, S. 5; Franzen 1995, S. 565; Keller 1998, S. 356).

2. Monetäre Transformation von Markenstärke zum Markenwert: Grundsätzlich besteht ein Trade-off zwischen einer finanzwirtschaftlichen eindimensionalen Markenbewertung und einer eher verhaltensorientierten mehrdimensionalen Markenbeurteilung (Sattler 1995, S. 678; Jenner 2001, S. 57; Kriegbaum 2001, S. 118ff.). Obwohl es interessante Versuche gibt, diese Trade-offs abzuschwächen - entweder wie beim Interbrand-Modell durch Multiplikatoren oder mit Hilfe von Brückenindikatoren (Kernstock et al. 2001, S. 35f.) - bleibt es ein idealtypischer Versuch, alle Einflussfaktoren auf den finanziellen Wert vollumfänglich und empirisch begründet zu ermitteln und zu gewichten.

3. Berücksichtigung von Markenausdehnungen: Die meisten Verfahren bewerten den Status quo einer Marke. Aus der Managementperspektive interessiert aber auch deren Erweiterungspotenzial, zumal zahlreiche Unternehmen dazu übergehen, gar keine neuen Marken mehr einzuführen, sondern ausschließlich vorhandene Marken auszudehnen (Sattler 1995, S. 668, 678). Das Transferpotenzial einer Marke ist als Realoption mitzubewerten (Jenner 2001, S. 60ff.): Bewertungskriterien hierfür sind Marktattraktivität, Wachstumsrate, Konkurrenzintensität sowie Ausmaß und Fit der Markenausdehnung (Jenner 2001, S. 60). Diese Aspekte werden häufig vernachlässigt, was dazu führt, dass ein zu niedriger Markenwert ausgewiesen wird. Letztlich gilt aber auch hier: Solche Realoptionen lassen sich zwar mithilfe komplexer Bewertungsmodelle der Optionspreistheorie bewerten, doch wird aufgrund der Vielzahl subjektiver Einschätzungen eine Scheingenauigkeit vorgetäuscht (Jenner 2001, S. 57). Alternativ bietet sich die Bewertung anhand von Expertenurteilen an, die allerdings keinen exakt bestimmbaren Wert liefert (Jenner 2001, S. 58).

4. Kontextbezogenheit der Bewertung: Die meisten Bewertungsverfahren versuchen, den Wert von Einzelmarken zu erfassen. In der Regel treten Unternehmen aber mit mehreren Marken am Markt auf, sodass eine umfassende Evaluation das gesamte 
Markensystem berücksichtigen müsste: Welche Synergien und Antinomien bestehen zwischen den Einzelmarken? Wie ist ihr Verhältnis zu einer etwaigen Dachmarke einzuschätzen? Dies unterstreicht wiederum die Unternehmensspezifität des Markenwerts: Die Ertragsquellen und somit auch der resultierende Wert einer Marke können variieren, je nachdem, welches Unternehmen die Marke in seinem Portfolio führt. Das erklärt letztlich, warum es einen - wenn auch in der Regel nicht effizienten - Markt für Marken (und somit auch für Kundenbeziehungen) gibt.

\subsection{Integration des Markenwerts in das Markencontrolling}

Welche handlungsorientierten Schlussfolgerungen können vor dem Hintergrund dieser Herausforderungen für die Messung des Markenwerts im Rahmen eines Markencontrollings gezogen werden? Das Konstrukt „Markenwert“ sollte in keinem Fall mit einer einzigen Treibergröße oder der Markenstärke verwechselt werden. Vielmehr sollte die Messung in jedem Fall mehrdimensional und unter Verwendung unterschiedlicher Verfahren erfolgen. Rein ökonomische Messverfahren helfen zwar zu ermitteln, wie sich ein Markenwert entwickelt, erklären diesen aber nicht (Esch/Andresen 1994, S. 219). Allerdings erreichen rein verhaltenswissenschaftliche Messungen nur Marketingexperten; für andere Führungskräfte und die Finanzmärkte sind sie zu wenig aussagekräftig. Die größte Herausforderung ist aber der unterschiedliche Zeithorizont: Der Aufbau von Marken benötigt Zeit und die meisten Unternehmen verfügen bei finanzwirtschaftlichen Aspekten nicht über die erforderliche Geduld. Folgende drei Möglichkeiten können als Handlungsanleitung für eine Methodenkombination dienen:

\section{Markenstärke als Basis für operative Entscheidungen}

Informationen über die zentralen Treiber der Markenstärke sind unerlässliche Voraussetzungen für fundierte Markenentscheide. Daher sind kriterienorientierte Messverfahren erforderlich, die die wichtigsten verhaltenswissenschaftlichen Größen sowie marktorientierte Kennzahlen abdecken. Wichtig ist dabei eine kontinuierliche (Zeitreihenanalysen), konkurrenz- und kundenorientierte Messung: Insbesondere die Differenzierung einer Marke auf der Basis kundenrelevanter Aspekte ist eine notwendige Voraussetzung für einen langfristigen Markterfolg (Aaker 1996, S. 328).

Als eine mögliche aggregierte Spitzenkennzahl empfiehlt sich ein mehrdimensionaler Markenstärkeindex. Ein Vorschlag hierzu ist das Brand-Rating-DreikomponentenModell (Spannagl 2001), das auf dem Brand-Trek-Modell von icon zur Messung der qualitativen Markenstärke basiert. Dieses wird mit einem diskontierten Preisabstand und einem „Brand Future Score“ kombiniert; letzterer soll das Markenpotenzial erfassen hinsichtlich Dehnung (Produktlinien-, Distributions-, Zielgruppen- und regionale Ausdehnung), Entwicklungstrend (Preis- und Mengenentwicklung, zukünftige Bedeutung) sowie Krisenanfälligkeit (rechtlicher Schutz, Missbrauchs- 
gefahr). Auch wenn die Fundierung der Kriterienauswahl und ihre Verknüpfung (Addition vs. Multiplikation) kritisch hinterfragt werden muss, so ist das Modell ein erster Versuch für eine integrierte Bewertung. Aufgrund der Auswahl- und Gewichtungsprobleme ist eine solche Spitzenkennzahl allerdings nur im Zusammenhang mit den Einzelgrößen einzusetzen, sodass eine Untersuchung der Ursache-WirkungsZusammenhänge weiterhin stattfinden kann.

\section{Messung des finanziellen Werts einer Einzelmarke in besonderen Fällen}

Vor dem Hintergrund besonderer Situationen erscheint es sinnvoll, den finanziellen Wert einer Einzelmarke zu berechnen - beispielsweise bei Entscheidungen über Markenverkäufe und -lizenzierungen sowie insbesondere über etwaige Markenausdehnungen.

Die auf den kriterienorientierten Verfahren basierenden Multiplikatormodelle eignen sich eher für stabile Geschäftsbereiche, während einkommensorientierte diskontierte Geldflussanalysen für neue Geschäftsfelder zu bevorzugen sind, in denen nicht auf umfangreiche Daten zurückgegriffen werden kann (Ward 1989). Andererseits sind letztere nicht besonders sinnvoll, wenn Kundenloyalität und Wechselkosten gering sind, die Innovationsgeschwindigkeit hoch ist und sich die Marke in einer frühen Phase des Lebenszyklus befindet (Srivastava/Shocker 1991, S. 21). Aufgrund der Bewertungsunsicherheiten sind Sensitivitätsanalysen unverzichtbar.

Des Weiteren besteht auch die Möglichkeit, den finanzwirtschaftlichen Markenwert in ein diagnostisches Marketingkennzahlensystem zu integrieren. Wenn die Messung im Rahmen von Zeitreihenanalysen regelmäßig und mithilfe der gleichen Messinstrumente erfolgt, kann diese Größe insbesondere im Konkurrenzvergleich ein Indiz für die langfristige Entwicklung einer Marke sein. Ein solcher finanzwirtschaftlicher Markenwert sollte aber aufgrund der messbedingten Volatilität niemals als Steuerungs-, sondern lediglich als Diagnosekennzahl dienen.

\section{Portfolioorientierte Messung der Performance von Mehrmarkenstrategien}

Die bisherigen Hinweise haben sich auf die Bewertung von Einzelmarken beschränkt. Letztlich wird aber der Wert jeder Marke auch durch das Markenportfolio beeinflusst. Bisher fehlen in der Marketingwissenschaft differenzierte Hinweise darauf, wie die Interaktion zwischen verschiedenen Marken zu bewerten ist. Lediglich erste Empfehlungen zum Management von Mehrmarkenstrategien (Meffert/Perrey 1998) sowie zum kennzahlenorientierten Mehrmarkencontrolling liegen bis dato vor. Aaker (1996, S. 239ff.) hat beispielsweise ein Rollenmodell entwickelt, in dem den einzelnen Marken eines Portfolios unterschiedliche Funktionen zukommen. Es ist zu erwarten, dass in Zukunft auch kriterienorientierte Bewertungsmodelle für Mehrmarkenstrategien entwickelt werden. Derzeit muss dazu sich eine entsprechende Bewertung noch auf relativ einfache Instrumente wie z.B. Markenportfolios und 
Wanderungsanalysen beschränken; ferner sind Analogien zu Geschäftsfeldportfolios möglich (Meffert 2000, S. 249ff.).

Die Schilderungen haben gezeigt, dass es „den“ Markenwert als absolute und valide Einzelgröße nicht gibt. Vielmehr sollten Marketingführungskräfte danach streben, sich diesem Konstrukt mit einem Methodenmix aus verhaltenswissenschaftlichen und finanzwirtschaftlichen Verfahren zu nähern, um daraus Handlungsempfehlungen ableiten zu können. Übergeordnete Markenstärkeindizes sind geeignet, wenn sie durch Einzelindikatoren erklärt werden. Der finanzwirtschaftliche Markenwert sollte dagegen allenfalls als kontinuierliche Diagnose-, nicht aber als Steuerungskennzahl eingesetzt werden; in gewissen Sondersituationen (beispielsweise bei der Veräußerung von Markenrechten) kommt ihm jedoch eine wichtige Funktion zu. Insgesamt unterstützt eine solche Quantifizierung die reflexive Komponente im Rahmen der Willensbildung, die aber niemals ausschließlich zu berücksichtigen ist.

\section{Messung der Wirkungen von Markenstärke und -wert}

Im Folgenden werden beispielhafte Ansätze zur Messung der Wirkungen von Markenstärke diskutiert, die einige der erläuterten Methoden kombinieren und/oder Erhebungen des Markenwertes und relevanter zugrunde liegender Dimensionen im Zeitverlauf ermöglichen.

Die Wirkungen des Markenwertes lassen sich mit komparativen Ansätzen (marken- bzw. marketingorientiert) oder durch Conjoint-Analysen (Kombination der beiden Ansätze) erheben. Bei den markenorientierten komparativen Ansätzen (Keller 2001, S. 1076f.) handelt es sich um Vergleichsexperimente, d.h., eine bestimmte Variable wird variiert und die Auswirkungen auf eine andere Variable werden untersucht. In diesem Fall werden unterschiedliche Marken analysiert, wobei jeweils eine Maßnahme des Marketingmix konstant gehalten wird. Als Beispiel hierfür lassen sich Blindtests anführen. Dabei wird den Konsumenten ein und dasselbe Produkt in neutraler Verpackung vorgelegt, wobei jeweils unterschiedliche Markennamen und -logos verwendet werden. Bei derartigen Tests lassen sich häufig signifikante Unterschiede zwischen den Markenbeurteilungen der Konsumenten je nach Kennzeichnung beobachten. Eine weitere Variante der markenorientierten komparativen Ansätze besteht darin, die Auswirkungen verschiedener Marken auf die Preisbereitschaft der Konsumenten zu untersuchen. Schließlich können auch Kommunikationsarten und -inhalte getestet werden. Bei diesem Verfahren werden die Konsumenten aufgefordert, beispielsweise eine Zeitungsanzeige oder einen Werbespot zu beurteilen. Dieser ist jeweils gleich gestaltet und enthält die gleichen Inhalte, wobei die Marken entsprechend variiert werden. 
Bei den marketingorientierten komparativen Ansätzen werden ebenfalls Vergleichsexperimente durchgeführt (Keller 2001, S. 1077f.). Hierbei werden die Auswirkungen unterschiedlicher Maßnahmen des Marketingmix analysiert, wobei jeweils die gleiche Marke betrachtet wird. Ein Verfahren besteht darin, Konsumenten zu ihrer Kaufbereitschaft für eine Marke bei unterschiedlichen Preisen zu befragen. Interessant wäre beispielsweise die Ermittlung von Preisober- und -untergrenzen für Haushaltsgeräte der Marke Miele. Hierbei könnte ermittelt werden, wie hoch der Rabatt beispielsweise für Waschmaschinen anderer Marken angesetzt werden müsste, damit die Konsumenten sich gegen die Qualitätsmarke Miele und für eine konkurrierende Marke entscheiden, oder welchen Preis die Konsumenten für die (subjektive) Qualität einer MieleWaschmaschine zu zahlen bereit wären. $\mathrm{Zu}$ den marketingorientierten komparativen Ansätzen zählt auch die Durchführung von Testmärkten.

Als dritter Ansatz zur Messung der Wirkungen des Markenwerts ist die ConjointAnalyse zu nennen (Keller 2001, S. 1078f.). Hierbei werden marken- und marketingorientierte komparative Ansätze durch den Einsatz multivariater Verfahren kombiniert. Somit werden sowohl mehrere Marken betrachtet als auch unterschiedliche Elemente des Marketingmix analysiert. Mit Hilfe der Conjoint-Analyse werden insbesondere auch Probleme bei der Erhebung von Zahlungsbereitschaften für Marken umgangen.

\section{Mehrdimensionale Ansätze des Markencontrollings}

Nachfolgend werden einige mehrdimensionale Ansätze des Markencontrollings beispielhaft vorgestellt (z.B. Keller 1998, S. 373ff.). Mit einem Brand Audit wird das Ziel verfolgt, möglichst umfassende Analysen sämtlicher Einflussgrößen zu liefern, die den Markenwert beeinflussen, um darauf basierend Hinweise für die strategische Markenführung ableiten zu können. Ein Brand Audit sollte regelmäßig oder zumindest sporadisch durchgeführt werden, um Informationen über die Markenentwicklung zu gewinnen (beispielsweise um zu überprüfen, ob bestimmte Assoziationen verstärkt wahrgenommen werden, nachdem sie im Marketing betont wurden). Bei einem Brand Audit sind sowohl die unternehmensinterne (,Welche Produkte werden unter der Marke angeboten?“, „Welchen Umsatz und welche Margen erzielen sie?“, „Wie wird die Marke kommuniziert?“ usw.) als auch die konsumentenorientierte Perspektive (,Bei welchen Gelegenheiten wird die Marke benutzt?“, „Welche Assoziationen verbinden Konsumenten mit der Marke?“, „Welche Marken werden ähnlich wahrgenommen?“ usw.) zu berücksichtigen, um ein umfassendes Bild von Performance und Wahrnehmung der Marke zu gewinnen.

Der so genannte Markenfilter von McKinsey (Braun et al. 2003, S. 19ff.) ist ein verhaltensorientierter Ansatz, um unterschiedliche Marken eines Unternehmens oder aber Konkurrenzmarken miteinander zu vergleichen. Der Markenfilter gliedert den Prozess 
von Kundenakquisition und -bindung für jedes Zielgruppensegment in die fünf Schritte Bekanntheit, Interesse, Versuch, Präferenz und Loyalität (vgl. Schaubild 8). Der Filter visualisiert dabei Schwachstellen im Kundenprozess und analysiert, an welcher Stelle im Benchmarkingvergleich besonders viele (potenzielle) Käufer oder Kunden verloren gehen. Der Markenfilter ist ein einfaches, auf Effektivität ausgerichtetes Instrument, das danach strebt, dem Top-Management Hinweise für den wirkungsvollen Einsatz (knapper) Marketingressourcen zu geben. Voraussetzung für dessen Anwendung sind allerdings zuverlässige Marktforschungsdaten; auch müssen die Zielsegmente klar abgegrenzt sein, um aussagekräftige Vergleiche zu ermöglichen. Für ein umfassendes Markenaudit muss der Markenfilter ferner durch einstellungsorientierte Verfahren ergänzt werden, die das Markenwissen differenzierter messen.

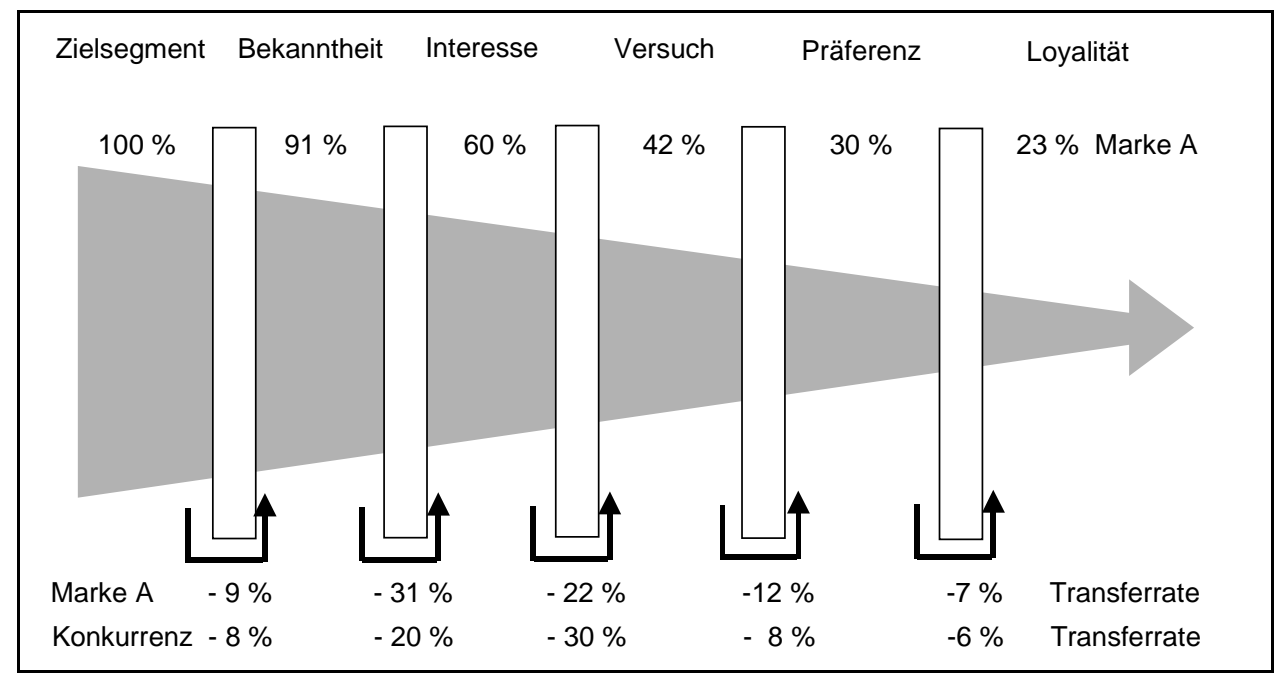

Schaubild 8: McKinsey-Markenfilter

(Quelle: In Anlehnung an Braun et al. 2003, S. 19)

Trackingstudien (Keller 1998, S. 380ff.) dienen primär dazu, Informationen über die Entwicklung von Marken sowohl aus unternehmensinterner als auch aus konsumentenorientierter Perspektive zu liefern. Im Gegensatz zu Brand Audits sind sie weniger umfangreich und konzentrieren sich auf die Analyse zentraler Größen; diese werden in regelmäßigen Abständen mittels standardisierter Befragungen erhoben bzw. intern ermittelt. Nachfolgend werden vier Methoden exemplarisch dargestellt.

1. Die Gesellschaft für Konsumforschung (GfK), Nürnberg stellt mit dem Brand ASessment System (BASS) (Högl/Hupp 2001) ein Instrument zur Verfügung, das ein mehrdimensionales Markencontrolling in regelmäßigen Abständen ermöglicht. Es 
basiert zum einen auf „Hard Facts“, d.h. dem realisierten Erfolg einer Marke am Markt, zum anderen auf „Soft Facts“, d.h. der Wahrnehmung der Marke durch die Konsumenten.

Zur Ermittlung des Markterfolgs werden Paneldaten herangezogen, die der regelmäßigen Bestimmung des Marktanteils einer Marke dienen. Der Markterfolg wird hierbei basierend auf der Käuferreichweite (Anzahl der Konsumenten, die die betreffende Marke im betrachteten Zeitraum mindestens einmal kaufen), auf der Anzahl der First Choice Buyer (Konsumenten, die die betreffende Marke im betrachteten Zeitraum als erstpräferierte Marke erwerben) und auf dem First Choice Value (Anteil der Käufe der First Choice Buyer am gesamten Umsatz der betreffenden Marke) ermittelt.

- Zur Erhebung der Wahrnehmung der Marke durch die Konsumenten werden regelmäßig Interviews mit Konsumenten durchgeführt, um Indikatoren zu erfassen, die die emotionale Haltung und die kognitiven Prozesse der Konsumenten gegenüber Marken widerspiegeln. Beispiele für derartige Indikatoren sind die wahrgenommene Qualität, die Markensympathie sowie die Bereitschaft zur Weiterempfehlung.

2. Eine methodisch ähnliche Lösung wurde vom schweizerischen Institut für Marktanalysen (IHA-GfK) mit dem Brand Health Check entwickelt. Dieser basiert ebenfalls sowohl auf Paneldaten als auch auf Informationen zu Konsumenteneinstellungen. Allerdings werden nicht die Daten des regulären Verbraucherpanels herangezogen, sondern es wird ein Testpanel mit 6.000 Haushalten bzw. 16.500 Personen genutzt. Dieses umfasst sowohl Fragen zum tatsächlichen Kauf- und Nutzungsverhalten von Marken als auch zu den zugrunde liegenden Markenbekanntheitsgraden und -einstellungen.

3. Der vom Schweizerischen Marktforschungsinstitut Demoscope konzipierte Market Radar eignet sich ebenfalls zur Durchführung von Tracking-Studien im Rahmen des Markencontrolling. Der Market Radar ist ein Softwarepaket, das mehrere Module und Funktionen zur Unterstützung von Marketingentscheidungen umfasst. Im Hinblick auf das Markencontrolling sind insbesondere die Darstellungen zur Segmentierung von Märkten und Positionierung von Marken auf Basis von LifestyleTypologien relevant. Der Lebensstil eines Konsumenten wird anhand seiner Meinungen, Einstellungen und Werte festgelegt. Persönliche Werthaltungen eignen sich besonders gut zur Entwicklung von Lifestyle-Typologien, da sie von kurzfristigen situativen Änderungen relativ wenig beeinflusst werden (Meffert 1998, S. 192). Innerhalb eines durch zwei Achsen (beispielsweise mit den Dimensionen „Außenvs. Innenorientierung" sowie ,progressive vs. konservative Grundhaltung") markierten Raums werden bestimmte Werthaltungen eingeordnet. Die Auswahl der Werthaltungen basiert auf einem Verfahren für Persönlichkeitstests, mit dem das Werteprofil eines Individuums erstellt wird. Auf Basis von Marktforschungsdaten lassen sich die Marken der betrachteten Produktkategorie in diesem Spektrum positionieren. 
Diese Analysen werden regelmäßig durchgeführt, sodass sich der Market Radar zum Brand Tracking eignet - beispielsweise zum Aufzeigen von Positionierungsveränderungen.

4. Die von der Firma Sinus Sociovision entwickelten Sinus Milieus liefern differenzierte Informationen über die Lebenswelten von Konsumenten (Sinus Sociovision 2000). Durch Analysen auf Basis der Sinus Milieus können Markenmanager spezifische Informationen über die Werthaltungen und Einstellungen ihrer Zielgruppen gewinnen, die über die Erhebung der üblichen soziodemographischen Daten hinausgehen. „Die Sinus Milieus fassen Konsumenten zusammen, die sich in Lebensauffassung und Lebensweise ähneln. Grundlegende Wertorientierungen gehen dabei ebenso in die Analyse ein wie Alltagseinstellungen zur Arbeit, zur Familie, zur Freizeit, zu Geld und Konsum. Sie rücken also den Menschen und das gesamte Bezugssystem seiner Lebenswelt ganzheitlich ins Blickfeld“ (Sinus Sociovision 2000, S. 2). Wichtigen gesellschaftlichen Trends und den dadurch ausgelösten Veränderungen wird durch eine regelmäßige Überarbeitung der Sinus Milieus Rechnung getragen. Somit können Markenmanager durch Image- und Markenkernanalysen zu unterschiedlichen Zeitpunkten beispielsweise ermitteln, welche gesellschaftlichen Trends die Werthaltungen ihrer Zielgruppe beeinflusst haben und wie sich die Position ihrer Marke verändert hat. Erkenntnisse aus der Trend- und Milieuforschung nach dem Ansatz von Sinus Sociovision eignen sich somit als Basis für TrackingStudien von Marken, insbesondere zur Analyse der Ursachen für die Markenwahrnehmung und Einstellungen der Konsumenten.

Ein weiterer mehrdimensionaler Ansatz des Markencontrolling ist die Marken-BalancedScorecard bzw. Brand Score Card (vgl. hierzu ausführlich Meffert/Koers 2001 sowie den Beitrag von Linxweiler/Brucker in Teil 7 dieses Handbuches). Diese dient dazu, die Markenstrategie mit Hilfe monetärer und nicht-monetärer Kenngrößen zu operationalisieren, um dadurch die Umsetzung der Markenführung zu optimieren.

\section{Markenspezifisches Marketingaccounting}

Ein markenspezifisches Marketingaccounting liefert insbesondere Informationsgrundlagen für Erfolgsbeiträge bestehender und künftiger Marken sowie die Erfolgswirkungen von Marketinginstrumenten. Dabei stehen insbesondere Deckungsbeitrags- bzw. Marktsegmentrechnungen im Mittelpunkt (Köhler 1993, S. 303ff.) - aus Markensicht vor allem die Produkt- bzw. Markenerfolgsrechnung (vgl. Schaubild 9). Deckungsbeitragsrechnungen können als Vorschaurechnung oder Ergebniskontrolle durchgeführt werden. Bei einer Vorschau ist das Prinzip der Veränderungsrechnung zu berücksichtigen: Nur jene Kosten sind in das Entscheidungskalkül einzubeziehen, die sich durch die Entscheidung für eine Handlungsalternative verändern (Köhler 1993, S. 287). Die Kosten 
werden in fixe und proportionale Bestandteile aufgeteilt und - ausgehend vom Nettoumsatz werden produkt-, produktgruppen-, markenspezifische Kosten stufenweise zugerechnet (Ehrmann 2001, S. 609ff.).

+ Theoretischer Markenumsatz (Normal- bzw. Nettopreis x Menge)

- Temporäre Preisaktionen der Marke

([Normalpreis - Aktionseinstandspreis] x Menge)

- Markenbezogene Werbebeitragszahlungen/-kostenzuschüsse, Listungsgebühren

sowie weitere auf Handelsstufe verlangte Sonderkonditionen (Trade Incentives)

- Selbstkosten (Costs of Goods sold) (ggf. auch Einstandspreis zzgl. Konfektionierungskosten für Spezialverpackungen, Displays sowie Warenhaus- und Transport kosten)

= Markendeckungsbeitrag nach Handelsaktionen

(Marginal Contribution less Trade Deals)

- Kosten von Verkaufsunterstützung (Vorverkäufe, Merchandising) (Sales Support)

= Markendeckungsbeitrag nach Handelsaktionen und Verkaufsunterstützung (Marginal Contribution less Trade Deals, less Sales Support)

- Relative Einzelkosten der Marke in der Periode (Gehalt der Markenmanager, spezifische Produkt- und Markenwerbung)

= Markendeckungsbeitrag nach Handelsaktionen/Verkaufsunterstützung/Marketing (Marginal Contribution less Trade Deals, less Sales Support, less Marketing)

Schaubild 9: Mehrstufige Markendeckungsbeitragsrechnung

(Quelle: In Anlehnung an Gmünder 2001, S. 839ff.)

Bei Kraft Foods wird beispielsweise der Deckungsbeitrag nach Handelsaktionen als absolute Zielgröße sowie als Zielgröße je Absatzmenge vorgegeben (Gmünder 2001, S. 841). Insbesondere bei Markenartikelherstellern mit indirekter Distribution empfiehlt sich auch eine weitergehende kunden- bzw. distributionskanalbezogene Berechnung. So werden den Kunden bzw. Distributionskanälen die Kosten für die Verkaufsunterstuitzung zugerechnet; die „Marginal Contribution less Trade Deals, less Sales Support“ dient daher bei Kraft Foods als zentrale Kennziffer zur Steuerung der Kunden (Gmünder 2001, S. 842).

Das Grundproblem von Deckungsbeitragsrechnungen besteht darin, dass der Erfolg eines einzelnen Produkts bzw. einer einzelnen Marke immer fiktiv ist, weil sich in den seltensten Fällen Markttransaktionen und Geschäftsbeziehungen auf isolierte Produkte oder Marken beziehen (Engelhardt/Günter 1988, S. 144). Daher sind insbesondere mehrdimensionale Deckungsbeitragsrechnungen ratsam. 
Die Prozesskostenrechnung als „,neueres“, aber auch aufwändigeres Instrument des internen Rechnungswesens strebt eine verursachergerechte Zurechnung der betrieblichen Gemeinkosten auf die Marken oder andere Marktsegmente an und hilft insbesondere bei der Identifikation von Kostentreibern (vgl. hierzu ausführlich Reckenfelderbäumer 2001, S. 650ff.). Eine Marktsegmentrechnung, die der Prozesskostenrechnung ähnelt, ist die Berechnung der direkten Produktprofitabilität (Tomczak/Lindner 1992; Köhler 1993, S. 305f.). Dabei wird danach gestrebt, ein genaueres Bruttoergebnis pro Artikeleinheit zu ermitteln, indem die Kosten beispielsweise über artikelgenaue Zeit-, Flächen- und Volumenmessungen geschlüsselt werden. Der Ansatz entspricht einer Teilkostenrechnung, die allerdings nicht nur proportionale, sondern - im Gegensatz zur klassischen Deckungsbeitragsrechnung - auch fixe Kosten, wie z.B. Personalkosten, aufteilt. Der Ausdruck „direkt“ ist somit nicht ganz korrekt und verstößt streng genommen auch gegen das genannte Prinzip der Veränderungsrechnung (Köhler 1993, S. 305); dennoch kann diese Rechnung wichtige Signal- und Steuerungsaufgaben - bezogen auf Engpasseinheiten - übernehmen.

Das Marketingaccounting liefert wichtige weitere Informationen für die Gestaltung der Marketinginstrumente im Rahmen der Markenführung. So unterstützt es beispielsweise die Preisfindung für Neuprodukte (wobei darauf hinzuweisen ist, dass Kosten hier eher eine Kontrollgröße sind; sie sind somit insbesondere für die Ermittlung von Preisuntergrenzen zentral). Dabei können progressive oder retrograde Kalkulationsmethoden wie das Target Costing eingesetzt werden (Ehrmann 2001, S. 594ff.). Ferner hilft es dabei, die Rationalität bei Sortimentsentscheidungen (Marken- oder Produkteliminationen, -selektionen oder -ergänzungen) sicherzustellen. Solche Entscheidungen sollten allerdings aufgrund der Verbundwirkungen in der Regel nicht ausschließlich auf der Basis von Kostenrechnungsinformationen fallen. Auch für die Gestaltung der markenbezogenen Kommunikation sowie die akquisitorische und physische Distributionspolitik liefert das Marketingaccounting wichtige Informationen: beispielsweise für die Gestaltung und Optimierung markenbezogener Kommunikationsbudgets, die Provisionsgestaltung des Außendienstes sowie die Optimierung der Logistik (Köhler 1993, S. 307ff.).

\section{Fazit}

Markencontrolling bedeutet, die Effektivität und Effizienz der Markenführung sicherzustellen. Dazu ist es erforderlich, sowohl markt-, konsumenten- bzw. verhaltenswissenschaftliche als auch kosten- und finanzwirtschaftliche Informationen für das Markenmanagement bereitzustellen. Des Weiteren muss die Willensdurchsetzung beispielsweise durch den Einsatz von Kenngrößen erleichtert werden. Kontrolle ist eine wichtige, wenn auch nicht die einzige Aufgabe des Markencontrolling: Auch sie muss wiederum verhaltenswissenschaftlich (z.B. Markenwissen) als auch finanzwirtschaftlich (z.B. 
Markenwert und Markenaccounting) erfolgen. Das Markencontrolling übernimmt eine zentrale Qualitätssicherungsfunktion der Markenführung, ohne jedoch institutionelle Entscheidungsgewalt zu beanspruchen.

\section{Literaturverzeichnis}

AAKER, D.A. (1991): Managing Brand Equity, Capitalizing on the Value of a Brand Name, New York u.a.

AAKER, D.A. (1996): Building Strong Brands, New York u.a.

Agarwal, M.K./RAO, V.R. (1996): An Empirical Comparison of Consumer-Based Measures of Brand Equity, in: Marketing Letters, Vol. 7, No. 3, S. 237-247.

BAUMGARTH, C. (2001): Markenpolitik. Markenwirkungen, Markenführung, Markenforschung, Wiesbaden.

BEKMEIER-FEUERHAHN, S. (1998): Marktorientierte Markenbewertung, Wiesbaden.

BEKMEIER-FEUERHAHN, S. (2001): Messung von Markenvorstellungen, in: Esch, F.-R. (Hrsg.), Moderne Markenführung, 3. Aufl., Wiesbaden, S. 1105-1122.

BIEL, A.L. (2001): Grundlagen zum Markenwertaufbau, in: Esch, F.-R. (Hrsg.), Moderne Markenführung, 3. Aufl., Wiesbaden, S. 61-90.

Braun, M./KopkA, U./TochtermanN, T. (2003): Promotions - ein Fass ohne Boden, in: Akzente, 27. Jg., Nr. 4, S. 16-23.

Cravens, K.S./Guilding, C. (1999): Strategic Brand Valuation: A Cross-Functional Perspective, in: Business Horizons, Vol. 42, No. 4, S. 53-60.

CRIMminS, J.C. (1992): Better Measurement and Management of Brand Value, in: Journal of Advertising Research, Vol. 32, No. 4, S. 11-19.

EHRmann, H. (2001): Marketingaccounting, in: Reinecke, S./Tomczak, T./Geis, G. (Hrsg.), Handbuch Marketingcontrolling, St. Gallen und Wien, S. 586-613.

ENGELHARDT, W.H./GÜNTER, B. (1988): Erfolgsgrößen im internen Rechnungswesen aus der Sicht der Absatzpolitik, in: Domsch, M./Eisenführ, F./Ordelheide, D./ Perlitz, M. (Hrsg.), Unternehmenserfolg. Planung, Ermittlung, Kontrolle, Wiesbaden, S. 141-155.

ESCH, F.-R. (2001): Kontrolle der Eigenständigkeit von Markenauftritten, in: Esch, F.-R. (Hrsg.), Moderne Markenführung, 3. Aufl., Wiesbaden, S. 1123-1138.

ESCH, F.-R./ANDRESEN, T. (1994): Messung des Markenwerts, in: Tomczak, T./Reinecke, S. (Hrsg.), Marktforschung, St. Gallen, S. 212-230.

EsCH, F.-R./Geus, P. (2001): Ansätze zur Messung des Markenwerts, in: Esch, F.-R. (Hrsg.), Moderne Markenführung, 3. Aufl., Wiesbaden, S. 1025-1057.

FRANZEN, O. (1995): Die praktische Nutzung der Markenbewertungssysteme, in: Markenartikel, 57. Jg., Nr. 12, S. 562-566.

GRUNERT, K.G. (1990): Kognitive Strukturen in der Konsumforschung, Wirtschaftswissenschaftliche Beiträge, Band 30, Heidelberg. 
GuILDING, C./PIKE, R. (1994): Brand Valuation: A Model and Empirical Study of Organisational Implications, in: Accounting and Business Research, Vol. 24, No. 95, S. 241-253.

GMÜNDER, P. (2001): Kundenspezifisches Marketingcontrolling in der Konsumgüterbranche, in: Reinecke, S./Tomczak, T./Geis, G. (Hrsg.): Handbuch Marketingcontrolling, St. Gallen und Wien, S. 836-845.

GÜLDENBERG, H.G./FrANZEN, O. (1992): Auditing auf der Basis von Markenwerten, in: Thexis, 9. Jg., Nr. 5, S. 37-43.

HerRmanN, A. (1998): Produktmanagement, München.

HöGL, S./HuPP, O. (2001): Markenbewertung mit dem Brand Assessment System, in: Markenartikel, 63. Jg., Nr. 4, S. 22-27.

HORVÁTH, P. (1998): Controlling, 7. Aufl., München.

JENNER, T. (2001): Markenführung in Zeiten des Shareholder-Value, in: Harvard Business Manager, 23. Jg., Nr. 3, S. 54-63.

KELlER, K.L. (1993): Conceptualizing, Measuring and Managing Customer-Based Brand Equity, in: Journal of Marketing, Vol. 57, No. 1, S. 1-22.

KELlER, K.L. (1998): Strategic Brand Management: Building, Measuring, and Managing Brand Equity, Upper Saddle River.

KELLER, K.L. (2001): Kundenorientierte Messung des Markenwerts, in: Esch, F.-R. (Hrsg.), Moderne Markenführung, 3. Aufl., Wiesbaden, S. 1059-1079.

KERnSTOCK, J./BrocKDORFF, B./ADERS, C./WIEDEMANN, F. (2001): Markenevaluation in der Konsumgüterindustrie und anderen markengetriebenen Branchen - Eine empirische Studie, St. Gallen u.a.

KÖHLER, R. (1993): Beiträge zum Marketing-Management. Planung, Organisation, Controlling, 3. Aufl., Stuttgart.

KRIEGBAUM, C. (2001): Markencontrolling. Bewertung und Steuerung von Marken als immaterielle Vermögenswerte im Rahmen eines unternehmenswertorientierten Controlling, München.

Kroeber-RIEL, W./WeInBerg, P. (1999): Konsumentenverhalten, 7. Aufl., München.

LEUTHESSER, L. (1988): Defining, Measuring, and Managing Brand Equity, Marketing Science Institute, Report Nr. 88-104, Cambridge (Mass.).

MEFFERT, H. (1982): 16 Meffert-Thesen zu Marketing und Controlling, in: Absatzwirtschaft, 25. Jg., Nr. 9, S. 100-107.

MEFFERT, H. (1999): Mehrmarkenstrategien - immer die beste Option?, in: Absatzwirtschaft, 42. Jg., Sondernummer Oktober, S. 82-87.

MEFFERT, H. (2000): Marketing. Grundlagen marktorientierter Unternehmensführung, Konzepte, Instrumente, Praxisbeispiele, 9. Aufl., Wiesbaden.

MEFFERT, H./KOERS, M. (2001): Integratives Markencontrolling auf Basis des BalancedScorecard-Ansatzes, in: Reinecke, S./Tomczak, T./Geis, G. (Hrsg.), Handbuch Marketingcontrolling, St. Gallen und Wien, S. 292-320.

MefFert, H./PERREY, J. (1998): Mehrmarkenstrategien - Ein Beitrag zum Management von Markenportfolios, Arbeitspapier Nr. 121 der Wissenschaftlichen Gesellschaft für Marketing und Unternehmensführung e.V., Münster. 
PAIVIO, A. (1971): Imagery and Verbal Processes, New York u.a.

RECKENFELDERBÄUMER, M. (2001): Prozesskostenrechnung im Marketing, in: Reinecke, S./Tomczak, T./Geis, G. (Hrsg.), Handbuch Marketingcontrolling, St. Gallen und Wien, S. 650-676.

REINECKE, S. (2003): Marketing Performance Management, St. Gallen.

RIEBEL, R. (1994): Einzelkosten- und Deckungsbeitragsrechnung, 7. Aufl., Wiesbaden.

RugE, H.-D. (1988): Die Messung bildhafter Konsumerlebnisse, Reihe Konsum und Verhalten, Band 16, Heidelberg.

SATTLER, H. (1995): Markenbewertung, in: Zeitschrift für Betriebswirtschaft, 65. Jg., Nr. 6, S. 663-682.

SATTLER, H. (1998): Beurteilung der Erfolgschancen von Markentransfers, in: Zeitschrift für Betriebswirtschaft, 68. Jg., Nr. 5, S. 473-495.

SAtTler, H. (2000): Markenbewertung, in: Albers, S./Herrmann, A. (Hrsg.), Handbuch Produktmanagement, Wiesbaden, S. 219-240.

SCHIELE, T.P. (1997): Markenstrategien wachstumsorientierter Unternehmen, Mannheim.

Simons, C.J./Sullivan, M.W. (1993): The Measurement and Determinants of Brand Equity: A Financial Approach, in: Marketing Science, Vol. 12, No. 1, S. $28-52$.

SINUS SOCIOVISION (2000): Kurzinformation zu den Sinus-Milieus 2000, Heidelberg.

SPANNAGL, J. (2001): Neuer Standard in der Markenbewertung, in: Markenartikel, 63. Jg., Nr. 5, S. 38-44.

SpIGgle, S./SEWAll, M.A. (1987): A Choice Sets Model of Retail Selection, in: Journal of Marketing, Vol. 51, No. 4, S. 97-111.

SrivastaVA, R.K./SHOCKER, A.D. (1991): Brand Equity: A Perspective on Its Meaning and Measurement, Technical Working Paper, Marketing Science Institute, Report Nr. 91-124, Cambridge (Mass.).

SRIVASTAVA, R.K./SHERVANI, T.A./FAHEY, L. (1998): Market-Based Assets and Shareholder Value: A Framework for Analysis, in: Journal of Marketing, Vol. 62, No. 1, S. 2-18.

TOMCZAK, T./LindNER, U. (1992): Keine Zukunft für DPR?, in: Thexis, 9. Jg., Nr. 4, S. 35-38.

TrommsdorfF, V./PAulssen, M. (2001): Messung und Gestaltung der Markenpositionierung, in: Esch, F.-R. (Hrsg.), Moderne Markenführung, 3. Aufl., Wiesbaden, S. 1139-1158.

WARD, K. (1989): Can Cash Flows on Brands Really be Capitalized?, in: Murphy, J. (Hrsg.), Brand Valuation. Establishing a True and Fair View, London.

WEBER, J. (1999): Einführung in das Controlling, 8. Aufl., Stuttgart.

WEBER, J./SCHÄFFER, U. (1998): Sicherstellung der Rationalität von Führung als Controllingaufgabe?, WHU-Forschungspapier Nr. 49, Vallendar.

WEBER, J./SCHÄFFER, U. (2001): Marketingcontrolling - Sicherstellung der Rationalität in einer marktorientierten Unternehmensführung, in: Reinecke, S./Tomczak, T./ Geis, G. (Hrsg.), Handbuch Marketingcontrolling, St. Gallen und Wien, S. 32-49. 
Wiedmann, K.-P. (1994): Strategisches Markencontrolling, in: Bruhn, M. (Hrsg.), Handbuch Markenartikel, Band 2, Stuttgart, S. 1305-1336.

YouNG \& RUBICAM (2000): Brand Asset Valuator, New York. 